\title{
Potential fluctuations and density of gap states in amorphous semiconductors
}

\author{
C. T. Chan* and Steven G. Louie \\ Department of Physics, University of California, Berkeley, California 94720 \\ and Materials and Molecular Research Division, Lawrence Berkeley Laboratory, Berkeley, California 94720 \\ J. C. Phillips \\ AT\&T Bell Laboratories, Murray Hill, New Jersey 07974
}

(Received 14 July 1986)

\begin{abstract}
The exponential-band-tail (Urbach-tail) problem for structurally disordered material is treated by a simple model in which quantum wells (used to model the effects of local disorder) trap states in the gap. The density of states in the gap is calculated and is shown to have approximately exponential behavior over a range of energy of physical interest. A direct relation is established between the Urbach-tail slope and the glass transition temperature. Agreement with experimentally determined Urbach-tail slopes is obtained for the Ge-Sn-Se glasses for reasonable parameters employed in the present model.
\end{abstract}

\section{INTRODUCTION}

Structural atomic disorder modifies electronic spectra, especially near band edges in amorphous semiconductors, where tails in the density of states (DOS) are produced below crystalline band edges. ${ }^{1}$ In insulators, tails are produced near exciton bands broadened statically by defects and thermally by lattice vibrations. These absorption tails have an exponential functional dependence $I(\omega)$ $\approx \exp (\hbar \omega / \sigma)$, as first noticed by Urbach. ${ }^{2}$ This exponential dependence has often been observed and in favorable cases has been found to hold for several orders of magnitude (up to four or five decades).

The problem of defining and solving in the tail region a quantum-mechanical model with large structural disorder is difficult even for drastically simplified Hamiltonians, whether the solution is obtained formally or numerically. . $3-6$ A closely related problem is the threshold behavior of the electrical conductivity of impurity bands at ultralow temperatures when the overlap of localized impurity states is varied through the metal-insulator transition. ${ }^{7}$ So far, neither an exact formal method nor a computational algorithm is known for solving these problems, and all the models that have been analyzed contain assumptions of one kind or another. ${ }^{8}$

Experimental evidence on the origin of Urbach tails in amorphous semiconductors mainly concerns the compositions and temperature dependence of $\sigma^{-1}$, the Urbach-tail slope. Toyozawa and co-workers have argued, ${ }^{1,3,9}$ from model Hamiltonians, that a white-noise model with a Gaussian-distributed site energy $\delta E$ can explain Urbach tails. If the crystalline bandwidth is of order $6 B$, and the Gaussian width of $\delta E$ is $W$, then they argue that $\sigma \approx S k_{B} T / 2 B$, where $k_{B}$ is Boltzmann's constant and $S$ (of order $B$ ) is the lattice relaxation energy such that $W^{2}=S k_{B} T$. The potential fluctuations are expected to be Gaussian because a large number of sources of randomness contribute to these fluctuations.
One of the drawbacks of the otherwise elegant Toyozawa models is that the convolution of crystalline band edges by Gaussians produces exponential behavior in the DOS only over relatively narrow energy ranges in three dimensions. For $|E|$ small, Gaussian fluctuations give

$$
\rho(E) \approx \exp (-|E| / \sigma)^{v}
$$

with

$$
v=2-\frac{d}{2}
$$

where $\rho(E)$ is the density of states and $E$ is measured either from the top of the valence band or from the bottom of the conduction band and $d$ is the dimensionality of the system. $^{4}$ For $|E|$ large, $\rho(E)$ is a Gaussian. Toyozawa argues that the exponential form holds only over a limited range of energy and results merely from an interplay between the bandwidth energy $B$ and the Gaussian fluctuation width $\boldsymbol{W}$.

For a glass at low $T$, the dominant static structural disorder corresponds to thermally induced disorder frozen in at $T=T_{g}$. In this paper, we attempt to model the effects of these static potential fluctuations by assuming that the non-bond-disrupting potential fluctuations are most important. This approximation should be valid in semiconductors with $\left|E-E_{0}\right| \ll B$ where $E_{0}$ is the Tauc ( $k$ non-conserving) optical band edge. ${ }^{1,10}$ We do not introduce an explicit model Hamiltonian, but instead treat the potential fluctuations in terms of quantum wells with a distribution of well size and depth given by a Boltzmannlike factor related to the total-energy changes resulting from the potential fluctuations. Our analysis leads to results which are of interest in connection with recent experiments on Urbach tails associated with grain boundaries ${ }^{11}$ and in selected binary and pseudobinary semiconductor glasses. ${ }^{12}$ 


\section{MODEL AND CALCULATIONS}

We consider a state of energy $\varepsilon$ in the gap near the top of the valence band as a state trapped by local disorder (thermal or static) which will be modeled by a quantum well ${ }^{13}$ of strength $V$ and radius $a$. The total energy needed for the formation of such a quantum well is of the order of the local change of electronic energy of the electrons inside the well:

$$
\Delta E \approx \int_{-\infty}^{E_{F}} \varepsilon \delta \rho(\varepsilon) d \varepsilon,
$$

where $\delta \rho$ is the change of density of states due to the perturbation induced by the quantum well and $E_{F}$ is the Fermi energy. The use of the difference in the sum of oneelectron energies to approximate total structural energy change has been justified by the Andersen force theorem ${ }^{14}$ and used successfully in applications to crystalline materials. ${ }^{14}$ We estimate the right-hand side of Eq. (3) by assuming that the density of states within the valence band is constant, and that the effect of the quantum well is a rigid shift of band energies of the electrons inside the well by an amount $V$. The total-energy change is then

$$
\Delta E \approx n V\left(\frac{4 \pi}{3} a^{3}\right) \xi^{\prime}=n V a^{3} \xi,
$$

where $n$ is the density of electrons in the material and $\xi=(4 \pi / 3) \xi^{\prime}$ is a dimensionless factor of order unity.

For given $V$ and $a$, the probability distribution for such quantum wells is given by the Boltzmann factor:

$$
P(V, a)=e^{-\Delta E / k_{B} T}=e^{-n V a^{3} \xi / k_{B} T},
$$

where $T$ is either the temperature for a pure crystal (thermal disorder) or a temperature near but above the glass transition temperature $T_{g}$ for a glass (static disorder). The density of states for states trapped by the quantum wells in the gap is given (up to a constant) by

$$
D(\varepsilon)=\int d V \int d a e^{\left(-n \xi / k_{B} T\right) V a^{3}} \delta(E(V, a)-\varepsilon)
$$

where $E(V, a)$ is the energy of defect states corresponding to a particular value of $V$ and $a$. The energy $\varepsilon$ is measured from the top of the valence band. In the present form, Eqs. (3) and (3') may be regarded as somewhat heuristic, and they can be tested by their ability to describe experimental data. ${ }^{12}$

We attempt a solution for a particle of negative effective mass, $m^{*}$, in a spherical well of depth $V$ and radius $a$, i.e.,

$$
V(r)= \begin{cases}V, & r<a \\ 0, & r>a\end{cases}
$$

Since it requires larger energy to create deeper wells, we will restrict ourselves to $l=0$ solutions (lowest energy for a given well) of the Schrödinger equation with sphericalwell potentials. For this simple case, the zero-angularmomentum state of energy $E$ is given by the equation ${ }^{15}$

$$
\alpha \cot (\alpha a)=-\beta,
$$

where

$$
\begin{aligned}
& \alpha=\left[\frac{2 m^{*}}{\hbar^{2}}(-V+E)\right]^{1 / 2}, \\
& \beta=\left[\frac{2 m^{*}}{\hbar^{2}}(-E)\right]^{1 / 2} .
\end{aligned}
$$

Using atomic Rydberg units and assuming $m^{*}=-m_{e}$, we have

$$
\begin{aligned}
& \alpha=(V-E)^{1 / 2}, \\
& \beta=E^{1 / 2} .
\end{aligned}
$$

Equation (5) may be written as

$D(\varepsilon)=\int d V \int d a \sum_{a^{\prime}}\left[\frac{\partial E}{\partial a}\right]_{a=a^{\prime}}^{-1} \delta\left(a-a^{\prime}\right) e^{\left(-n \xi / k_{B} T\right) V a^{3}}$,

where $a^{\prime}$ are all values of the well depth satisfying Eq. (7) given $\varepsilon$ and $V$ (or $\beta$ and $\alpha$ ) and are given by

$$
a^{\prime}=\frac{1}{\alpha}\left[m \pi-\cot ^{-1} \frac{\beta}{\alpha}\right),
$$

where $m$ are positive integers (since $a>0$ ) and $\cot ^{-1}(\beta / \alpha)$ is taken to be within the range $[0,(\pi / 2)]$.

It is straightforward to show that

$$
\left(\frac{\partial E}{\partial a}\right)^{-1}=\frac{1+\frac{\beta}{\alpha}\left[m \pi-\cot ^{-1} \frac{\beta}{\alpha}\right]}{2 \alpha^{2} \beta} \text {. }
$$

Hence we have

$$
\begin{aligned}
& D(\varepsilon)=\sum_{m(\geq 1)} \int_{\varepsilon}^{V_{\max }} d V\left|\frac{1+\frac{\beta}{\alpha}\left[m \pi-\cot ^{-1} \frac{\beta}{\alpha}\right]}{2 \alpha^{2} \beta}\right| \\
& \times \exp \left[\frac{-n \xi}{k_{B} T} V \frac{1}{\alpha^{3}}\left[m \pi-\cot ^{-1} \frac{\beta}{\alpha}\right]^{3}\right] .
\end{aligned}
$$

Because of the $m^{3}$ factor in the exponential, we may take only the $m=1$ component and obtain

$$
\begin{aligned}
& D(\varepsilon)=\int_{\varepsilon}^{V_{\max }} d V\left|\frac{1+\frac{\beta}{\alpha}\left[\pi-\cot ^{-1} \frac{\beta}{\alpha}\right]}{2 \alpha^{2} \beta}\right| \\
& \times \exp \left[\frac{-n \xi}{k_{B} T} V \frac{1}{\alpha^{3}}\left[\pi-\cot ^{-1} \frac{\beta}{\alpha}\right)^{3}\right] .
\end{aligned}
$$

Upper and lower bounds have been set for $V$ in the integral. This is because of the following.

(i) $V$ has to be greater than $\varepsilon$ in order for a bound state of energy $\varepsilon$ to exist and hence the lower limit. 
(ii) For an arbitrary small $a$, there always exists a $V$ large enough such that a given $\varepsilon$ is a solution of Eq. (7). However, we know that in a crystal, $a$ cannot be arbitrary small if $V$ is large. Thus, there is a cutoff $a=a_{\min }$ with a corresponding $V_{\max }$ which is related to $a_{\min }$ via Eq. (7). We expect that $a_{\min }$ is approximately a typical bond length which is $\approx \mathrm{a}$ few atomic units. Now changing to the variable

$$
y=\frac{\beta}{\alpha}=\left(\frac{\varepsilon}{V-\varepsilon}\right)^{1 / 2},
$$

we have

$$
\begin{aligned}
D(\varepsilon)=\frac{1}{\sqrt{\varepsilon}} \int_{y_{0}}^{\infty} & d y \frac{1+y\left(\pi-\cot ^{-1} y\right)}{y} \\
& \times \exp \left[-\Gamma \frac{1}{\sqrt{\varepsilon}}\left(\frac{1}{y^{2}}+1\right) y^{3}\left(\pi-\cot ^{-1} y\right)^{3}\right],
\end{aligned}
$$

where $y_{0}$ is given by the solution of

$$
\beta a_{\min }=y_{0}\left(\pi-\cot ^{-1} y_{0}\right),
$$

and

$$
\Gamma \equiv \frac{n \xi}{k_{B} T} .
$$

It is shown in the Appendix that $D(\varepsilon)$ given by Eq. (16) can be approximated analytically by

$$
\begin{aligned}
D(\varepsilon) \approx & \frac{1}{\Gamma}\left[\frac{1+\beta a_{\min }}{\beta^{2} a_{\min }^{2}\left[3+\left(3+y_{0}^{-2}\right) \beta a_{\min }\right]}\right] \\
& \times \exp \left[-\frac{\Gamma}{\sqrt{\varepsilon}}\left(\beta a_{\min }\right)^{3}\left(1+y_{0}^{-2}\right)\right] .
\end{aligned}
$$

Since $\beta=\sqrt{\varepsilon}$, we have

$$
\begin{aligned}
\ln D(\varepsilon) \approx & -\Gamma a_{\min }^{3}\left(1+y_{0}^{-2}\right) \varepsilon \\
& +\ln \left(\frac{1}{\Gamma} \frac{1+\sqrt{\varepsilon} a_{\min }}{\varepsilon a_{\min }^{2}\left[\left(3+y_{0}^{-2}\right) \sqrt{\varepsilon} a_{\min }+3\right]}\right),
\end{aligned}
$$

and hence

$$
\ln D(\varepsilon) \approx-\Gamma a_{\min }^{3}\left(1+y_{0}^{-2}\right) \varepsilon+O(\ln (\varepsilon)) .
$$

The second term is small and varies slowly with $\varepsilon$. As far as the slope of $\ln D(\varepsilon)$ is concerned, the first term dominates and we may write

$$
\ln D(\varepsilon) \approx-\Gamma a_{\min }^{3}\left(1+y_{0}^{-2}\right) \varepsilon .
$$

We first compare the approximate expression in Eq. (20) with values of $D(\varepsilon)$ determined directly from Eq. (16) by numerical integration for particular values of the parameters. We are interested in the energy range 0.01 $\mathrm{Ry}<\varepsilon<0.1 \mathrm{Ry} . a_{\min }$ is the smallest potential well in the glass and is taken to be $\sim 2$ a.u. Taking a glass transition temperature $T_{g} \approx 600 \mathrm{~K}, \xi \approx 1$, and $n \approx 0.03$ a.u., ${ }^{-3}$ we have $\Gamma \approx 10$. Using these values for the parameters, we have integrated Eq. (16) numerically for the energy range of interest. The results are presented in Fig. 1 together

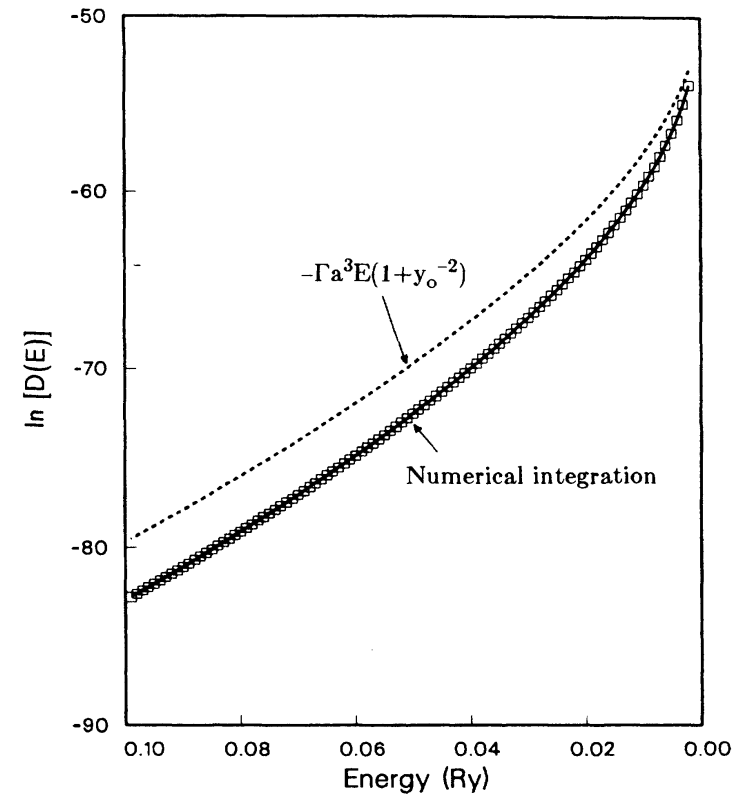

FIG. 1. Comparison of numerically integrated density of states with analytic approximations. The squares are determined from Eq. (16) by direct numerical integration. Equation (20) is plotted as the solid line (excellent agreement with numerical integration). Equation (22) is plotted as the dashed line. The parameters used are $a=2$ a.u. and $\Gamma=10\left(\text { a.u. }{ }^{3} \mathrm{Ry}\right)^{-1}$.

with those from the approximate analytic expression in Eq. (20). The parameter $y_{0}$ in Eq. (20) is obtained by solving Eq. (17). It can be seen that Eq. (20) almost agrees exactly with the numerical data. Results from Eq. (22) are also presented in Fig. 1. Comparison of these curves shows that the second term of Eq. (20) is small, and Eq. (22) gives a good approximation to the energy dependence of the density of states.

From Fig. 1, we see that for $\varepsilon>0.04 \mathrm{Ry}, \ln D(\varepsilon)$ is quite linear with respect to $\varepsilon$. In Figs. 2 and 3 , we plot numerically integrated values of $\ln D(\varepsilon)$ for different values of $\Gamma$ and $a_{\min }$. Essentially the same behavior is observed. The approximate linear behavior of $\ln [D(\varepsilon)]$ can be seen as follows. By Eq. (15),

$$
1+y_{0}^{-2}=\frac{V\left(\varepsilon, a_{\min }\right)}{\varepsilon},
$$

where $V\left(\varepsilon, a_{\min }\right)$ is the depth of a well of radius $a$, trapping a (lowest-energy) state with energy $\varepsilon$. Equation (22) may also be written as

$$
\begin{aligned}
\ln D(\varepsilon) & \approx-\Gamma a_{\min }^{3} V\left(\varepsilon, a_{\min }\right) \\
& =-\frac{n \xi}{k_{B} T} a_{\min }^{3} V\left(\varepsilon, a_{\min }\right) .
\end{aligned}
$$

$V\left(\varepsilon, a_{\min }\right)$ does not scale linearly with respect to $\varepsilon,{ }^{16}$ but for the range of energy in which we are interested, the deviation from linearity is small, as can be seen from the Figs. $1-3$. The right-hand side of Eq. (23) is just the ex- 


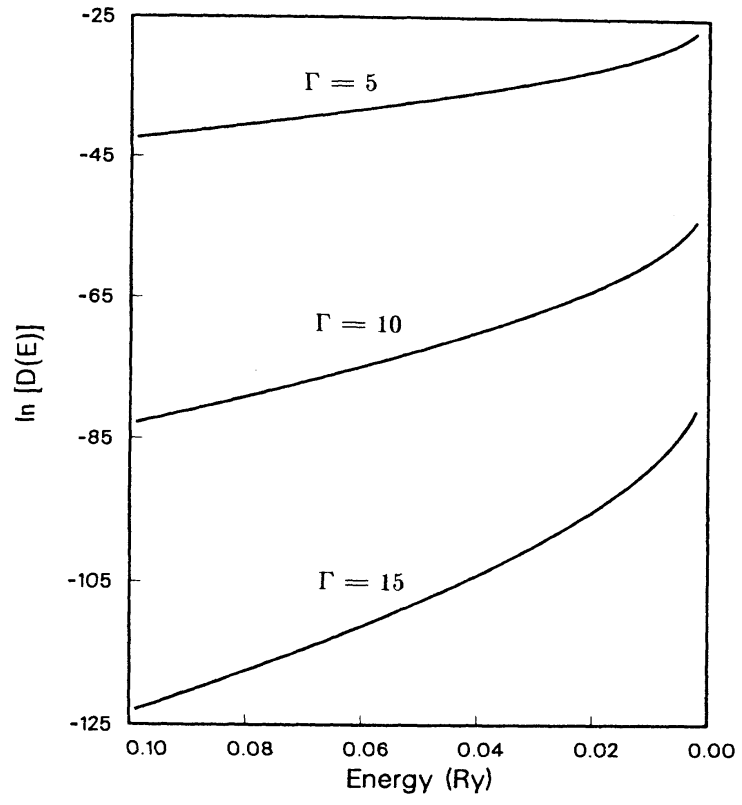

FIG. 2. Plot of the natural logarithm of the density of states $(\ln [D(E)])$ as determined from Eq. (16) by numerical integration against energy $(E)$ for different values of $\Gamma$. The well radius parameter $a=2$ a.u. $\Gamma$ is in units of $\left(\mathrm{a} \cdot \mathrm{u} \cdot{ }^{3} \mathrm{Ry}\right)^{-1}$.

ponential of the Boltzmann factor in Eq. (4) with $a=a_{\min }$. Thus, we see that the approximate exponential behavior of the density of states is essentially a consequence of the Boltzmann factor. For glasses, $\Gamma$ in Eq. (22) is determined by the relation $\Gamma=n \xi / k_{B} T_{g}$. Thus the model gives a direct correlation between the glass transi-

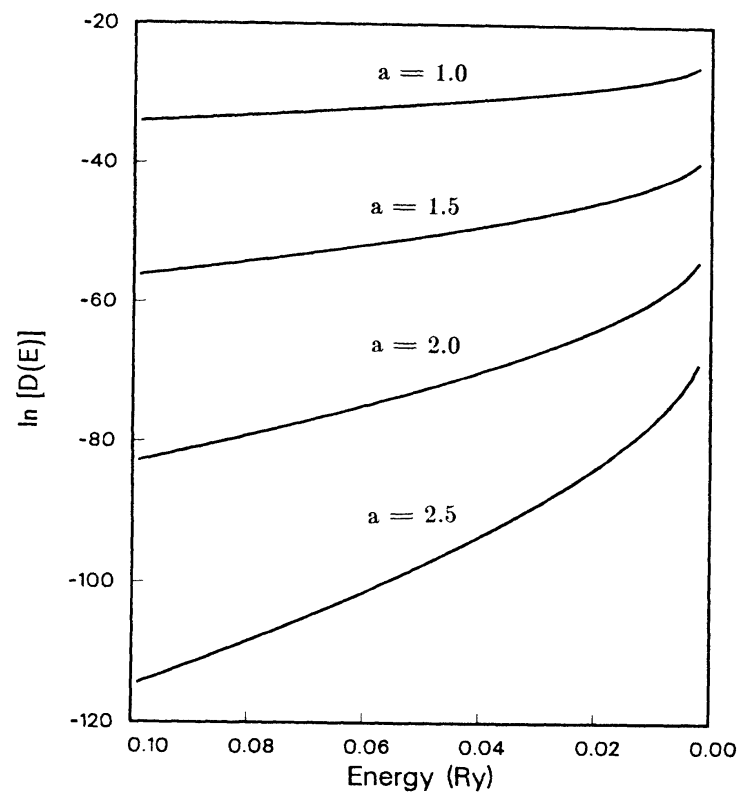

FIG. 3. Plot of the natural logarithm of the density of states $(\ln [D(E)])$ as determined from Eq. (16) by numerical integration against energy $(E)$ for different values of the well radius parameter $a$ (in a.u.). The parameter $\Gamma=10\left(\text { a.u. }{ }^{3} \mathrm{Ry}\right)^{-1}$. tion temperature $T_{g}$ and the experimental slope of the Urbach tail. The $a^{3}$ factor in the Eq. (3') means that the wells with the smallest radius commensurate with physical restrictions need the least energy to form and thus these wells with minimum radius $\left(a_{\min }\right)$ dominate the problem.

\section{DISCUSSION}

Two recent experiments have yielded theoretically significant results on Urbach tails in disordered semiconductors. Werner and Peisl ${ }^{11}$ have studied the density of states $N_{s s}(E)$ on grain-boundary surfaces of polycrystalline Si by two independent methods. They fitted their results to

$$
N_{s s}(E)=N_{0} \exp \left[-\frac{\Delta E}{E_{0}}\right]
$$

for both conduction and valence bands. $\Delta E$ in Eq. (24) is taken to be $\Delta E=E_{c}-E$ and $\Delta E=\left|E_{v}\right|-|E|$ for positive and negative energies $E$ from midgap, respectively. It was found that $E_{0}=49$ and $69 \mathrm{meV}$ for the conductionand valence-band electrons, respectively. Exponential behavior is observed over more than three decades. For two dimensions, exact exponential behavior is expected from Gaussian potential fluctuations [see Eq. (1)], so these data do not test the limitations of the Gaussian model for three dimensions. ${ }^{16}$

The second experiment, which is of greater theoretical interest, is the composition dependence of the Urbach slope $\sigma(x)$ in $\mathrm{Sn}_{x} \mathrm{Ge}_{1-x} \mathrm{Se}_{2.5}$ glasses. Previous studies of $\sigma(x)$ in $\mathrm{As}_{x} \mathrm{Se}_{1-x}$ and $\mathrm{Ge}_{x} \mathrm{Se}_{1-x}$ glasses showed that qualitatively $\sigma \sim k_{B} T_{g}$, where $T_{g}$ is the glass transition temperature, as expected from general considerations. ${ }^{1}$ However, the actual composition dependences of $\sigma(x)$ and $T_{g}(x)$ did not agree quantitatively. This was explained ${ }^{17}$ by the observation that varying $x$ in these binary alloys means varying the proportions of local building blocks [chain segments $\mathrm{Se}_{n}$, pyramids $\mathrm{As}\left(\mathrm{Se}_{1 / 2}\right)_{3}$, and tetrahedra $\left.\mathrm{Ge}\left(\mathrm{Se}_{1 / 2}\right)_{4}\right]$. Therefore, it was suggested ${ }^{17}$ that pseudobinary alloys like $\mathrm{Sn}_{x} \mathrm{Ge}_{1-x} \mathrm{Se}_{2}$ containing fixed proportions of building blocks of varying mechanical strength [Sn $\left(\mathrm{Se}_{1 / 2}\right)_{4}$ versus $\left.\mathrm{Ge}\left(\mathrm{Se}_{1 / 2}\right)_{4}\right]$ should be studied. These studies have shown a strong correlation, with $\sigma(x) \approx 1.5 k_{B} T_{g}(x)$ over the range $0 \leq x \leq 0.6$. This range goes from an overconstrained glass network $(x=0)$ to ideally constrained $(x=0.4)$ to underconstrained $(x=0.6)$ as described theoretically ${ }^{18}$ and confirmed by Mössbauer site ${ }^{19}$ and Raman bond ${ }^{20}$ studies. This is the first time such a close relationship between $\sigma$ and $T_{g}$ has been established.

The linear relation between $\sigma$ and $T_{g}$ is reproduced in the present model [Eq. (22)] if the structural disorder is assumed to arise from fluctuations frozen in at $T=T_{g}$. Using the parameter values $\Gamma=10$ (corresponding to $\left.T_{g}=600 \mathrm{~K}, n=0.03, \xi=1\right)$, and $a_{\min }=2$ a.u., we find that for $\varepsilon \geq 0.05 \mathrm{Ry}, 1 / \sigma=\partial \ln [D(\varepsilon)] / \partial \varepsilon \approx 200 \mathrm{Ry}^{-1}$, or $\sigma=68 \mathrm{meV}$. This gives $\sigma / k_{B} T_{g} \approx 1.3$, in good agreement with the value of $1.25 \pm 0.1$ found in the stoichiometric chalcogenide glasses ${ }^{21,22} g-\mathrm{As}_{2} \mathrm{Se}_{3}$ and $g-\mathrm{GeSe}_{2}$. The larger values which are found for other compositions are discussed elsewhere. ${ }^{23}$ 
Matrix element effects have been neglected in the estimation of $\sigma$. The optical transition density, $F(\varepsilon)$, has the same kernel as Eq. (5) weighted by $a^{-3}$ to account approximately for the photoexcitation oscillator strength ${ }^{3}$ with initial states localized in a volume of approximately $a^{3}$. In this case, $\ln F(\varepsilon)$ has the same expression as $\ln D(\varepsilon)$ in the form of Eq. (21) except that the second term is slightly different. In fact, to the same order of approximation as in Eq. (20), it can readily be shown that

$$
\ln F(\varepsilon)=\ln D(\varepsilon)-3 \ln a_{\min },
$$

where we use Eq. (20) for $\ln D(\varepsilon)$ in Eq. (25). Thus $\ln F(\varepsilon)$ has the same energy derivative as $\ln D(\varepsilon)$ and there is no difference whether we estimate $\sigma$ from $D(\varepsilon)$ or $F(\varepsilon)$.

In summary, our model assumes that the Urbach tails observed in amorphous semiconductors are produced by potential fluctuations frozen in at the glass transition temperature $\left(T_{g}\right)$. The probability distribution of these fluctuations depends on their formation energy and is governed by the Boltzmann factor. As a consequence, a proportionality relationship between $\sigma$ and $k_{B} T_{g}$ is found with a constant factor near 1.3. Experimental identification of this factor and its calculation contributes significantly to understanding the physical origin of the defects responsible for Urbach tails. ${ }^{23}$

After this paper was submitted for publication, a paper employing assumptions similar to ours appeared. ${ }^{24}$ However, in that paper, the ratio $\sigma / k_{B} T_{g}$ is not determined. In another recently published paper, ${ }^{25}$ exponential band tails have been obtained with "correlated potential fluctuations." The presentation is mathematically more elaborate, but the derivation of the exponential form of the density of states relies essentially on the same short-range potential (square well or Gaussian) that we use. No connection is made between their parameters $V_{\text {rms }}$ and $\varepsilon_{L}$ and the defect freezing temperature $T_{g}$, nor can we see a simple way to make such a connection, except through derivations similar to those given here.

\section{ACKNOWLEDGMENT}

This work was supported by National Science Foundation Grant No. DMR-83-19024 and by the Director, Office of Energy Research, Office of Basic Sciences, Materials Sciences Division of the U.S. Department of Energy under Contract No. DE-AC03-76SF00098.

\section{APPENDIX}

We show in this appendix that the integral in Eq. (16) can be approximated by Eq. (19). We first consider an in- tegral of the form

$$
I(\varepsilon)=\int_{\varepsilon}^{\infty} P(x) e^{-\Gamma x} d x
$$

with $P(x)$ a repeatedly differentiable function. By repeatly integrating by parts, one obtains

$$
I(\varepsilon)=\frac{P(\varepsilon)}{\Gamma} e^{-\Gamma \varepsilon}\left(1+\sum_{n=1} \frac{1}{\Gamma^{n}} \frac{P^{(n)}(\varepsilon)}{P(\varepsilon)}\right),
$$

where $P^{(n)}(x)$ denotes the $n$th derivative of $P(x)$ with respect to $x$.

Now, writing Eq. (16) in the form

$$
D(\varepsilon)=\frac{1}{\sqrt{\varepsilon}} \int_{y_{0}}^{\infty} d y \zeta(y) e^{-(\Gamma / \sqrt{\varepsilon}) \eta(y)},
$$

where

$$
\zeta(y)=\frac{1}{y}\left[1+y\left(\pi-\cot ^{-1} y\right)\right],
$$

and

$$
\eta(y)=\left(\frac{1}{y^{2}}+1\right) y^{3}\left(\pi-\cot ^{-1} y\right)^{3},
$$

and $y_{0}$ is given by Eq. (17). By changing the integration variable to $\eta$, we have

$$
D(\varepsilon)=\frac{1}{\sqrt{\varepsilon}} \int_{\eta_{0}}^{\infty} d \eta f e^{-(\Gamma / \sqrt{\varepsilon}) \eta},
$$

where $\eta_{0}=\eta\left(y_{0}\right)$ and $f=\zeta(y)(d \eta / d y)^{-1}$. For the range of parameters we are interested in (see text for discussion), $\Gamma / \sqrt{\varepsilon}>30 \gg 1$, we may use just the first term in Eq. (A2) and obtain

$$
D(\varepsilon) \approx \frac{1}{\Gamma} f\left(\eta_{0}\right) e^{-(\Gamma / \sqrt{\varepsilon}) \eta_{0}},
$$

with

$$
\eta_{0}=\left(\frac{1}{y_{0}^{2}}+1\right) y_{0}^{3}\left(\pi-\cot ^{-1} y_{0}\right)^{3}=\left(\frac{1}{y_{0}^{2}}+1\right) \beta^{3} a_{\text {min }}^{3},
$$

and straightforward manipulation leads to

$$
f\left(\eta_{0}\right)=\frac{1+\beta a_{\min }}{\left(\beta a_{\min }\right)^{2}\left[3+\left(3+y_{0}^{-2}\right) \beta a_{\min }\right]} .
$$

Substituting Eqs. (A7) and (A8) in Eq. (A6) gives us Eq. (19) in the text.
*Present address: Department of Physics, Ames Laboratory, Iowa State University, Ames, IA 50011.

${ }^{1}$ S. Abe and Y. Toyozawa, J. Phys. Soc. Jpn. 50, 2185 (1981).

${ }^{2}$ F. Urbach, Phys. Rev. 92, 1324 (1953).

${ }^{3}$ M. Schreiber and Y. Toyozawa, J. Phys. Soc. Jpn. 51, 1528 (1982); 51, 1537 (1982); 51, 1544 (1982).

${ }^{4}$ B. I. Halperin and M. Lax, Phys. Rev. 148, 722 (1966).

${ }^{5}$ M. H. Cohen, C. M. Soukoulis, and E. N. Economou, J. NonCryst. Solids 77\& 78, 171 (1985).
${ }^{6}$ C. M. Soukoulis, M. H. Cohen, and E. N. Economou, Phys. Rev. Lett. 53, 616 (1984)

${ }^{7}$ M. A. Paalanen, T. F. Rosenbaum, G. A. Thomas, and R. N. Bhatt, Phys. Rev. Lett. 48, 1284 (1982); G. A. Thomas, Y. Ootuka, S. Katsumoto, S. Kobayashi, and W. Sasaki, Phys. Rev. B 25, 4288 (1982).

8J. C. Phillips, Solid State Commun. 47, 191 (1983); Philos. Mag. 47, 407 (1983); O. N. Dorokhov, Solid State Commun. 51,381 (1984) 
${ }^{9}$ H. Sumi and Y. Toyozawa, J. Phys. Soc. Jpn. 31, 342 (1971).

10J. Tauc, Mater. Res. Bull. 5, 721 (1970).

${ }^{11}$ J. Werner and M. Peisl, Phys. Rev. B 31, 6881 (1985).

${ }^{12}$ A. Ksendzov, F. H. Pollak, J. Ihm, J. C. Phillips, and G. P. Espinosa, Bull. Am. Phys. Soc. 30, 480 (1985).

${ }^{13}$ Quantum well models have been applied to amorphous materials in other contexts. See, for example, M. H. Brodsky, Solid State Commun. 36, 55 (1980).

${ }^{14} \mathrm{~A}$. R. Mackintosh and O. K. Andersen, in Electrons at the Fermi Surface, edited by M. Springford (Cambridge University Press, Cambridge, England, 1980); H. L. Skriver, Phys. Rev. B 31, 1909 (1985).

${ }^{15}$ L. I. Schiff, Quantum Mechanics, 3rd ed. (Mcgraw-Hill, New York, 1971), Chap. 4.

${ }^{16}$ For $\sqrt{\varepsilon} a$ small, $V(\varepsilon, a)$ can be approximated by

$$
V(\varepsilon, a) \approx \varepsilon+\frac{\sqrt{\varepsilon}}{a}+\frac{\pi^{2}}{8 a^{2}}+\frac{\pi}{8 a^{2}}\left(16 \sqrt{\varepsilon} a+\pi^{2}\right)^{1 / 2},
$$

and hence for very small $\varepsilon, \ln D(\varepsilon)$ has a leading term that scales as $\sqrt{\varepsilon}$.
${ }^{17}$ J. Ihm, Solid State Commun. 53, 293 (1985); J. Phys. C (to be published).

18J. C. Phillips, Solid State Commun. 47, 203 (1983); J. C. Phillips and M. F. Thorpe, Solid State Commun. 53, 699 (1985).

${ }^{19}$ M. Stevens, J. Grothaus, P. Boolchand, and J. G. Hernandez, Solid State Commun. 47, 199 (1983).

${ }^{20} \mathrm{~K}$. Murase and T. Fukunaga, in Proceedings of the 17th International Conference on the Physics of Semiconductors, edited by J. D. Chadi and W. A. Harrison (Springer, New York, 1985), p. 943; J. C. Phillips, Phys. Rev. B 31, 8157 (1985).

${ }^{21}$ R. S. Sussman, I. G. Austin, and T. M. Searle, J. Phys. C 8, L182 (1975).

${ }^{22}$ H. Oheda, Jpn. J. Appl. Phys. 18, 1973 (1979).

${ }^{23}$ A. Ksendov, F. H. Pollak, G. P. Espinosa, and J. C. Phillips (preceding paper), Phys. Rev. B 35, 2740 (1987).

${ }^{24}$ Y. Bar-Yam, D. Adler, and J. D. Joannopoulos, Phys. Rev. Lett. 57, 467 (1986).

${ }^{25}$ S. John, C. Soukoulis, M. H. Cohen, and E. N. Economou, Phys. Rev. Lett. 57, 1777 (1986). 